\title{
MicroRNA-96 promotes the proliferation of colorectal cancer cells and targets tumor protein p53 inducible nuclear protein 1 , forkhead box protein 01 (FOXO1) and FOXO3a
}

\author{
FENG GAO $^{1}$ and WENHUI WANG ${ }^{2}$
}

Departments of ${ }^{1}$ Anorectal Surgery and ${ }^{2}$ Oncology, The People's Hospital of Weifang, Weifang, Shandong 261041, P.R. China

Received January 8, 2014; Accepted September 18, 2014

DOI: $10.3892 / \mathrm{mmr} .2014 .2854$

\begin{abstract}
MicroRNAs (miRNAs) are a conserved class of small, endogenous, non protein-coding RNA molecules that are capable of regulating gene expression at post-transcriptional levels and are involved in diverse cellular processes, including cancer pathogenesis. It has previously been reported that miRNA-96 (miR-96) is overexpressed in human colorectal cancer (CRC). However, the underlying mechanism of miR-96 regulation in CRC remains to be elucidated. In the present study, miR-96 was confirmed to be upregulated in CRC tissues by reverse transcription quantitative polymerase chain reaction. MTT assay, colony formation assay and cell cycle analysis revealed that miR-96 overexpression led to increased tumor cell viability, colony formation ability and cell cycle progression. By contrast, inhibition of miR-96 resulted in the suppression of cell proliferation. It was also demonstrated that miR-96 reduced the messenger RNA and protein expression levels of tumor protein p53 inducible nuclear protein 1 (TP53INP1), forkhead box protein O1 (FOXO1) and FOXO3a, which are closely associated with cell proliferation. A luciferase reporter assay indicated that miR-96 inhibited luciferase intensity controlled by the 3'UTRs of TP53INP1, FOXO1 and FOXO3a. In conclusion, the results of the present study demonstrated that miR-96 contributed to CRC cell growth and that TP53INP1, FOXO1 and FOXO3a were direct targets of miR-96, suggesting that miR-96 may have the potential to be used in the development of miRNA-based therapies for CRC patients.
\end{abstract}

\section{Introduction}

MicroRNAs (miRNAs) are a conserved class of small, endogenous, non-coding single-stranded RNA molecules that have

Correspondence to: Dr Wenhui Wang, Department of Oncology, The People's Hospital of Weifang, 151 Guangwen Road, Weifang, Shandong 261041, P.R. China

E-mail: wangwenhui2592@163.com

Key words: microRNA, miR-96, cell proliferation, colorectal cancer the capacity to regulate gene expression at post-transcriptional levels by binding to complementary sequences in the 3' untranslated region (3'UTR) of the target messenger RNA (mRNA), leading to mRNA degradation or translational repression $(1,2)$. Evidence demonstrates that miRNAs participate in diverse biological processes, including developmental timing, cell growth, apoptosis and patterning of the nervous system (3). Through the detection and localization of miRNAs in genomic regions, Calin et al (4) revealed that numerous miRNAs are located in fragile sites and genomic regions associated with cancer, for example miR-15a and miR-16, implying that abnormal miRNA expression may be closely associated with cancer pathogenesis. Subsequent studies confirmed that miRNA expression is frequently deregulated in numerous types of cancer. miR-122, a liver-specific miRNA, is downregulated in hepatocellular carcinoma, resulting in the suppression of cell migration and proliferation and the induction of cell apoptosis by targeting AKT3 (5). Upregulated miR-30b/30d contributes to melanoma cell invasion and migration by targeting polypeptide $N$-acetylgalactosaminyltransferase 7, leading to the progression of metastasis (6). Therefore, miRNAs are important molecules involved in the pathogenesis of numerous types of cancer and have potential as key biomarkers for cancer diagnosis or prognosis (1).

Colorectal cancer (CRC) is one of the most prevalent malignancies worldwide and remains the second leading cause of malignancy-associated mortality, with one-third of patients succumbing to this disease (7). CRC often occurs due to lifestyle and increasing age, though a minority of the population have a greater susceptibility due to genetic mutations of tumor suppressors, oncogenes or the miRNA network $(8,9)$. With the development of novel therapeutic strategies for patients with $\mathrm{CRC}$, incidence has declined in the United States of America, although incidence is still increasing in certain countries (10). Previous studies have indicated that numerous miRNAs are differentially expressed in human CRC tissues, compared with those of adjacent non-cancerous tissues by microarray assay and reverse transcription quantitative polymerase chain reaction (RT-qPCR). These miRNAs include miR-96, miR-135b, miR-31 and miR-183, which are also upregulated in colorectal cancer tissues $(11,12)$. The functions of miR-135b, miR-31 and miR-183 in colorectal carcinogenesis indicated that these miRNAs promoted CRC growth and metastasis-associated traits by functioning as oncogenes (13-15). Therefore, further 
study was required in order to elucidate the roles and regulatory mechanisms of miR-96 in CRC.

The present study aimed to confirm whether miR-96 was overexpressed in CRC and evaluate the effects of miR-96 overexpression in CRC. miR-96 expression in 20 CRC tissues and corresponding non-cancerous tissues was determined using RT-qPCR. Functional studies, including MTT assay, colony formation assay and cell cycle progression were used to analyze the effects of miR-96 overexpression. The present study additionally aimed to identify the target genes of miR-96 in CRC using western blot analysis and luciferase assay. The results of the present study may aid the development of miRNA-based therapies for the treatment of CRC.

\section{Materials and methods}

Patient tissue samples. Twenty CRC tissues and corresponding non-cancerous tissues were acquired from patients primarily diagnosed with CRC by pathological confirmation and immunohistochemical staining at the People's Hospital of Weifang (Weifang, China). The samples were obtained during surgery. Consent was obtained from all patients for tissue sample collection and the study was approved by the Ethics Committee of the People's Hospital of Weifang (Weifang, China). Following collection, samples were stored at $-80^{\circ} \mathrm{C}$ prior to miR-96 expression analysis.

Cell culture and transfection. The human CRC cell lines SW480 and SW620 (American Type Culture Collection, Manassas, VA, USA) were cultured in RPMI-1640 medium (Invitrogen Life Technologies, Carlsbad, CA, USA) supplemented with $10 \%$ fetal bovine serum (FBS; Gibco-BRL, Invitrogen Life Technologies, Carlsbad, CA, USA). The cells were maintained in a humidified incubator with $5 \% \mathrm{CO}_{2}$ at $37^{\circ} \mathrm{C}$ and digested for passage using 0.1\% trypsin (Jiufeng Company, Beijing, China). miR-96 mimics, miR-96 mimics control (mimics-ctrl), miR-96 inhibitor and miR-96 inhibitor control were synthesized by Ribobio Co., Ltd (Guangzhou, China). Cells were transfected using Lipofectamine $^{\mathrm{TM}} 2000$ reagent (Invitrogen Life Technologies) according to the manufacturers' instructions.

$R N A$ isolation and $R T-q P C R$. Total RNA was extracted using TRIzol reagent (Qiagen, Hilden, Germany) from tissues and transfected cells according to the manufacturers' instructions. RNA concentration and quality was measured and $500 \mathrm{ng}$ of RNA was used for reverse transcription (RT). Moloney murine leukemia virus reverse transcriptase (Takara Bio, Inc., Otsu, Japan) was used for the complementary DNA (cDNA) synthesis. For the cDNA synthesis of miR-96, an miR-96 RT primer was used and U6 small nuclear RNA was used as an internal control. For the cDNA synthesis of target genes, the primer Oligo(dT) was used and $\beta$-actin was used as an internal control. Following completion of the RT reaction, the cDNA was diluted, prepared for PCR using a SYBR Premix Ex TaqTM (Perfect Real Time; Takara Bio, Inc.) and analyzed using an ABI 7300 Real-Time PCR System (Applied Biosystems Life Technologies, Foster City, CA, USA). PCR was performed according to the following conditions: $95^{\circ} \mathrm{C}$ for $30 \mathrm{sec}$, followed by 40 cycles at $95^{\circ} \mathrm{C}$ for $5 \mathrm{sec}$ and $60^{\circ} \mathrm{C}$ for $31 \mathrm{sec}$. The stem loop RT primers were designed according to the protocol described in a previous study (16) and the PCR primers were designed using Primer Express ${ }^{\circledR} 3.0$ software (Life Technologies, Grand Island, NY, USA). The RT and PCR primers were as follows: miR-96 RT primer, 5'-CTAACTGGTGTCGTGGAGTCGGCAATTCAGTTGAGCAAAAATG-3' and forward, 5'-ACACTCCAGCTGGGTTTGGCACTAGCACAT-3; U6 RT primer, 5'-CTCAACTGGTGTCGTGG AGTCGGCAATTCAGTTGA GAAAATATGGAAC-3' and forward, 5'ACACTCCAGCTGG GGTGCTCGCTTCGGCAGCACA-3'; $\beta$-actin forward, 5'-CTCCATCCTGG CCTCGCTGT-3' and reverse, 5'-GCTGTCACCTTCACCGTT CC-3'; tumor protein p53 inducible nuclear protein 1 (TP53INP1) forward, 5'-CTGTGCATAACTCCTGCCCT-3' and reverse, 5'-AACAATGAATATGCTGCCCC-3'; forkhead box protein O1 (FOXO1) forward, 5'-GAGTGGATGGTCAA GAGCGT-3' and reverse, 5'-TTCCTTCATTCTGCACACGA-3'; FOXO3a forward, 5'-CTACGAGTGGATGGTGCGTT-3' and reverse, 5'-TCTTGCCAGTTCCCTCATTC-3'.

MTT assay. Cell viability was determined by MTT assay. Briefly, transfected cells were seeded into 96-well plates and $10 \mu \mathrm{l}$ MTT (concentration, $5 \mathrm{mg} / \mathrm{ml}$; Sigma-Aldrich, St Louis, MO, USA) was added into $100 \mu \mathrm{l}$ medium at indicated time-points. The cells were incubated with MTT for $\sim 4 \mathrm{~h}$ at $37^{\circ} \mathrm{C}$, followed by removal of MTT and addition of $150 \mu \mathrm{l}$ DMSO. Following incubation with DMSO for $10 \mathrm{~min}$ in the dark, absorbance was measured at $570 \mathrm{~nm}\left(\mathrm{~A} 570_{\mathrm{nm}}\right)$ with a microplate reader (Biorad168-1000XC; Bio-Rad Laboratories, Hercules, CA, USA).

Colony formation assay. Transfected cells were seeded at a density of 200 cells/well into 12 -well plates for the colony formation assay. The medium was replaced every three days until the majority of the colonies contained $>50$ cells. The colonies were washed with phosphate-buffered saline (PBS), fixed with $4 \%$ formalin and stained with $5 \%$ crystal violet (Jinglai Company, Shanghai, China) for $\sim 15 \mathrm{~min}$. Finally, the colonies were imaged (Nikon-D610; Nikon Corporation, Tokyo, Japan) and counted.

Cell cycle analysis. Cell cycle analysis was performed using propidium iodide (PI) staining and flow cytometric analysis. The transfected cells were starved for $24 \mathrm{~h}$, followed by the addition of medium containing 10\% FBS for a further $24 \mathrm{~h}$. The cells were subsequently trypsinized and resuspended in cold PBS (0.1\% FBS). Following centrifugation at $228 \mathrm{x}$ g for $5 \mathrm{~min}$, the cells were washed twice with cold PBS (0.1\% FBS) and fixed in $70 \%$ ethanol for a minimum of one day. Following fixation, the cells were washed with cold PBS (0.1\% FBS) and the cell pellets were resuspended in $500 \mu \mathrm{l}$ PI solution [50 $\mu \mathrm{g} / \mathrm{ml}$ PI (Dingguo Biotechnology Co., Ltd., Beijing, China) from $50 \mathrm{X}$ stock solution $(2.5 \mathrm{mg} / \mathrm{ml}), 0.1 \mathrm{mg} / \mathrm{ml}$ RNase A and $0.05 \%$ Triton X-100] for $\sim 40 \mathrm{~min}$ at $37^{\circ} \mathrm{C}$. Finally, $3 \mathrm{ml}$ cold PBS was added and the cells were centrifuged at $513 \mathrm{x} g$ for $5 \mathrm{~min}$. The cell pellets were resuspended in $500 \mu \mathrm{l}$ PBS for flow cytometry (BD FACSCalibur ${ }^{\mathrm{TM}}$ Cell Sorting system; BD Biosciences, Franklin Lakes, NJ, USA) .

Western blot analysis. Western blot analysis was used to evalute TP53INP1, FOXO1 and FOXO3a protein expression in the transfected cells. Briefly, the cells were collected and 
lysed with radioimmunoprecipitation assay buffer $(50 \mathrm{~mm}$ Tris, $\mathrm{pH} 7.4,150 \mathrm{~mm} \mathrm{NaCl}, 1 \%$ nonyl phenoxypolyethoxylethanol, $0.5 \%$ sodium deoxycholate and $0.1 \%$ SDS) for $10 \mathrm{~min}$ on ice. Following centrifugation at $8,000 \times \mathrm{g}$ for $30 \mathrm{~min}$ at $4^{\circ} \mathrm{C}$, the supernatant was removed and the protein concentration was measured by bicinchoninic acid assay. $50 \mu \mathrm{g}$ protein was analyzed using 10\% SDS-PAGE. Following gel separation, the proteins were transferred into the polyvinylidene difluoride membrane (Dingguo Biotechnology Co., Ltd.) and blocked in 5\% milk. Rabbit polyclonal TP53INP1 antibody (ab9775; dilution, 1:1,000; Abcam, Cambridge, UK), rabbit monoclonal FOXO1 antibody (ab52587, 1:1,000 dilution; Abcam) and rabbit polyclonal FOXO3a antibody (ab47409; dilution, 1:1,000; Abcam) were used as primary antibodies. The horseradish peroxidase-conjugated goat monoclonal to rabbit immunoglobulin $\mathrm{G}$ was used as the secondary antibody (Sanying Biotechnology Inc., Wuhan, China). The bound antibodies were detected using Enhanced Chemiluminescence Plus Western Blotting Detection system (GE Healthcare, Little Chalfont, UK).

Luciferase reporter assay. The direct interaction between miRNA and its target mRNA was determined by luciferase reporter assay. The binding site of miR-96 in the 3'UTR of target mRNA was cloned into the pmirGLO Dual-Luciferase miRNA Target Expression Vector (Promega Corp., Madison, WI, USA) according to the manufacturer's instructions. The mutant binding sites were generated using Quikchange Lightning SiteDirected Mutagenesis kit (Agilent, Technologies, Inc., Santa Clara, CA, USA) according to the manufacturer's instructions. Cells were subsequently co-transfected with miRNA and the wild-type or mutant 3'UTR for luciferase assay. Following transfection for $48 \mathrm{~h}$, the cells were harvested for protein extraction. Luciferase intensity was examined using the Dual Luciferase Reporter Gene Assay kit (Beyotime Institute of Biotechnology, Haimen, China) according the manufacturer's instructions. Renilla luciferase intensity was used an internal control.

Statistical analysis. Values are presented as the mean \pm standard variation of three independent experiments. The differences between groups were examined by two paired Student's t-test (GraphPad version 5.0; GraphPad Software, Inc., La Jolla, CA, USA) and $\mathrm{P}<0.05$ was considered to indicate a statistically significant difference between values.

\section{Results}

miR-96 is upregulated in CRC tissues. Based on the results of previous studies, which indicated deregulated miRNA expression in CRC tissues and adjacent non-cancerous tissues as indicated by microarray assay $(11,12)$, miR-96 expression levels were examined using RT-qPCR in 20 paired CRC tissues and corresponding non-cancerous tissues (Fig. 1). The results demonstrated miR-96 was overexpressed in the majority of the $20 \mathrm{CRC}$ tissues and revealed that the mean expression levels were $\sim 1.5$-fold greater than those in the corresponding non-cancerous tissues, suggesting that the high-level expression of miR-96 may be correlated with CRC pathogenesis.

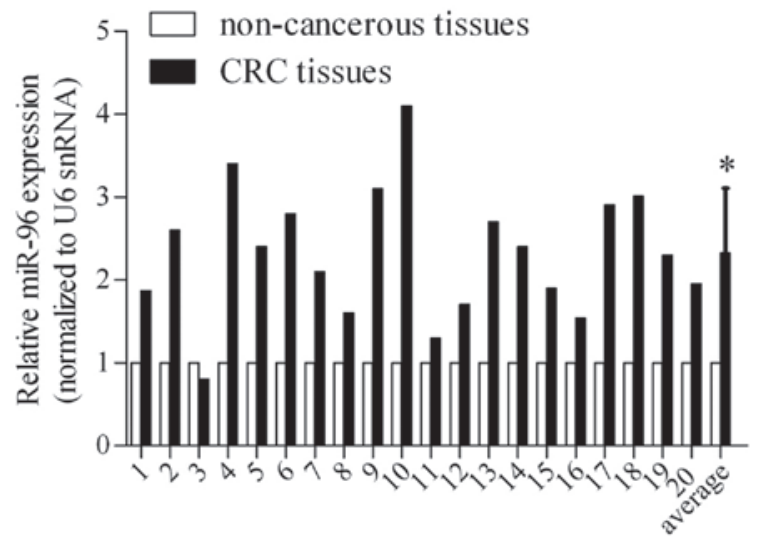

Figure 1. miR-96 is upregulated in CRC tissues. miR-96 expression was analyzed in 20 paired CRC tissues and corresponding non-cancerous tissues using reverse transcription quantitative polymerase chain reaction. U6 snRNA was used as an internal control. The miR-96 levels in non-cancerous tissues were normalized to $1 .{ }^{*} \mathrm{P}<0.05$ vs. non-cancerous tissues. miR, microRNA; CRC, colorectal cancer; snRNA, small nuclear RNA.

miR-96 overexpression promotes $C R C$ cell proliferation. To investigate the functional significance of deregulated miR-96 expression in the CRC cellular process, ectopic expression of miR-96 was achieved in SW480 and SW620 cells by transient transfection with miR-96 mimics (Fig. 2A). Cell viability was investigated at the indicated time-points by MTT assay. As indicated in Fig. 2B, it was demonstrated that ectopic miR-96 expression increased SW480 cell viability by $\sim 15 \%$, compared with that of the mimics ctrl-transfected cells. Ectopic miR-96 expression had the same effect on SW620 cell viability. A colony formation assay was performed to determine the roles of miR-96 in CRC-cell colony formation. It was discovered that miR-96 increased the number of colonies by $\sim 50$ and $\sim 80 \%$ in SW480 and SW620 cells, respectively (Fig. 2C), compared to that of the mimics ctrl group. In order to examine whether the contribution of miR-96 to cell growth was associated with cell cycle progression, the cell cycle was analyzed using PI staining. The results, shown in Fig. 2D, indicated that the number of miR-96-transfected cells at $\mathrm{G}_{1}$ phase was lower, whereas the number at $\mathrm{S}$ phase was higher in comparison to the numbers of cells transfected with mimics ctrl at each phase. These results suggested that miR-96 contributed to $\mathrm{G}_{1} / \mathrm{S}$ phase transition and cell proliferation.

Inhibition of miR-96 results in the suppression of CRC cell proliferation. To investigate whether miR-96 expression was essential for CRC cell growth, endogenous miR-96 expression was inhibited via transient transfection with an miR-96 inhibitor. The reduction in expression of miR-96 caused by the miR-96 inhibitor was confirmed by RT-qPCR (Fig. 3A). An MTT assay indicated that the inhibition of miR-96 led to a $\sim 20 \%$ reduction in cell viability (Fig. 3B). Accordingly, the number of colonies in SW480 and SW620 cells was also reduced due to the inhibition of miR-96 (Fig. 3C). Cell cycle analysis revealed that miR-96 inhibition resulted in a block of cell cycle progression at the $G_{1}$ phase transition, leading to the inhibition of cell proliferation. These results demonstrated a significant role for miR-96 in CRC pathogenesis. 

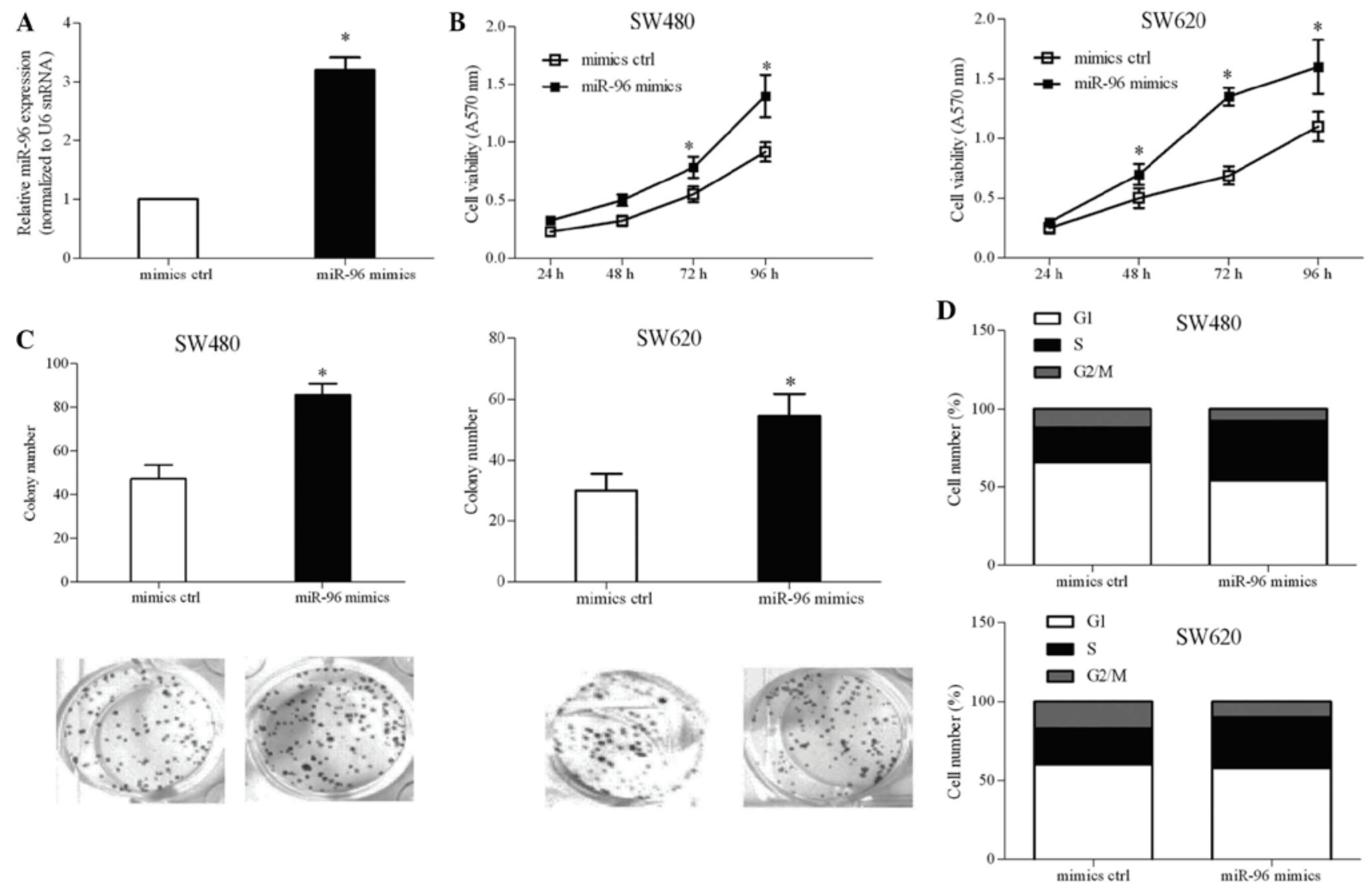

Figure 2. miR-96 promotes cell growth and proliferation in SW480 and SW620 cells. Cells were transiently transfected with miR-96 mimics or control and RNA was subsequently isolated for functional studies. (A) Reverse transcription polymerase chain reaction was performed for the analysis of miR-96 expression. U6 snRNA was used as an internal control. (B) Cell viability was measured by MTT assay at various time-points following transfection. (C) Transfected cells were subjected to colony formation assay. The graph represents the number of colonies. The image displays the colonies stained with crystal violet. (D) Cell cycle was analyzed using propidium iodide staining for the analysis of cell proliferation. Data are from three independent experiments and values are presented as the mean \pm standard deviation. ${ }^{*} \mathrm{P}<0.05$ vs. control group. miR, microRNA; snRNA, small nuclear RNA.
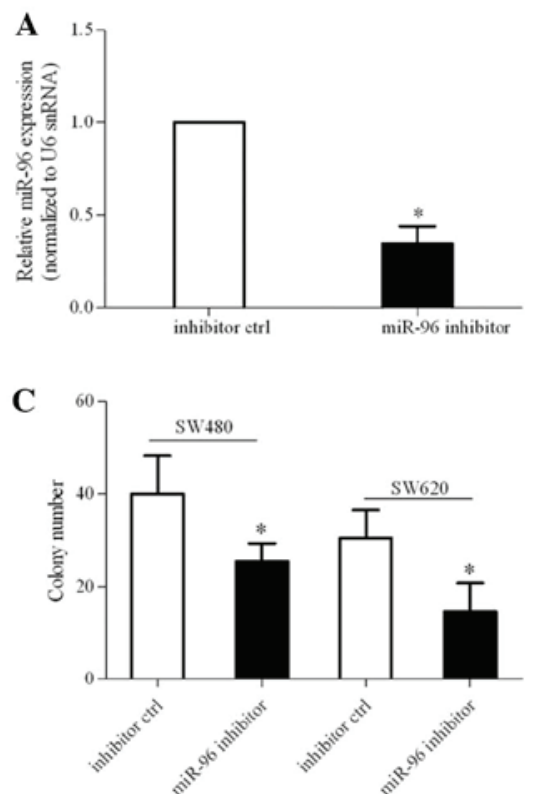

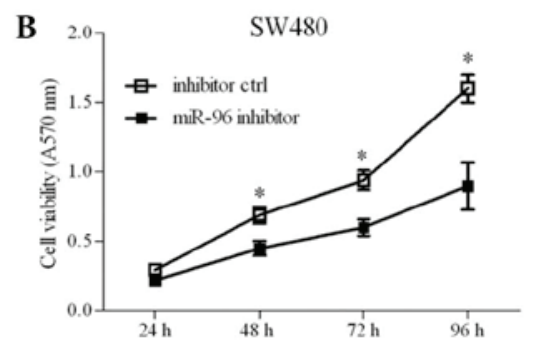

D

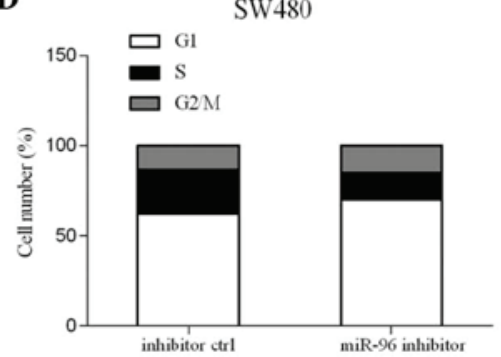

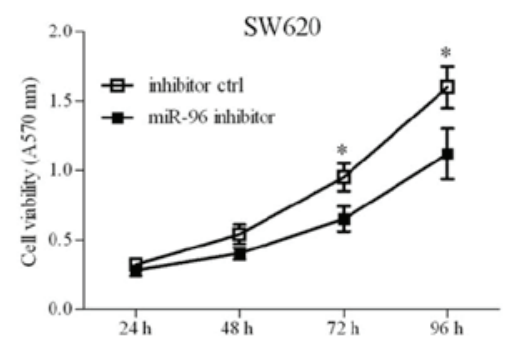

SW620

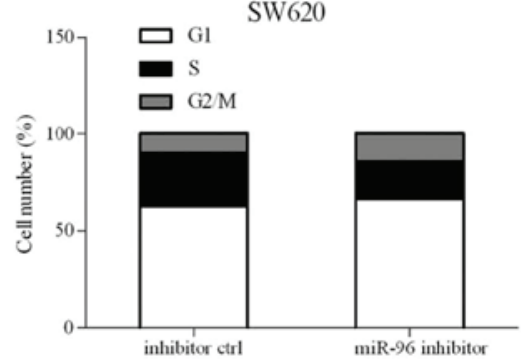

Figure 3. Inhibition of miR-96 suppresses cell growth and proliferation. Cells were transiently transfected with miR-96 inhibitor or control and RNA was subsequently isolated for functional studies. (A) Reverse transcription quantitative polymerase chain reaction was performed for the analysis of miR-96 expression. U6 snRNA was used as an internal control. (B) Cell viability was measured by MTT assay at various time-points following transfection. (C) Transfected cells were subjected to colony formation assay. The graph represents the number of colonies. (D) Cell cycle progression was analyzed using propidium iodide staining for the analysis of cell proliferation. Data are from three independent experiments and values are presented as the mean \pm standard deviation. ${ }^{*} \mathrm{P}<0.05$ vs control group. miR-96, micro RNA-96; snRNA, small nuclear RNA. 

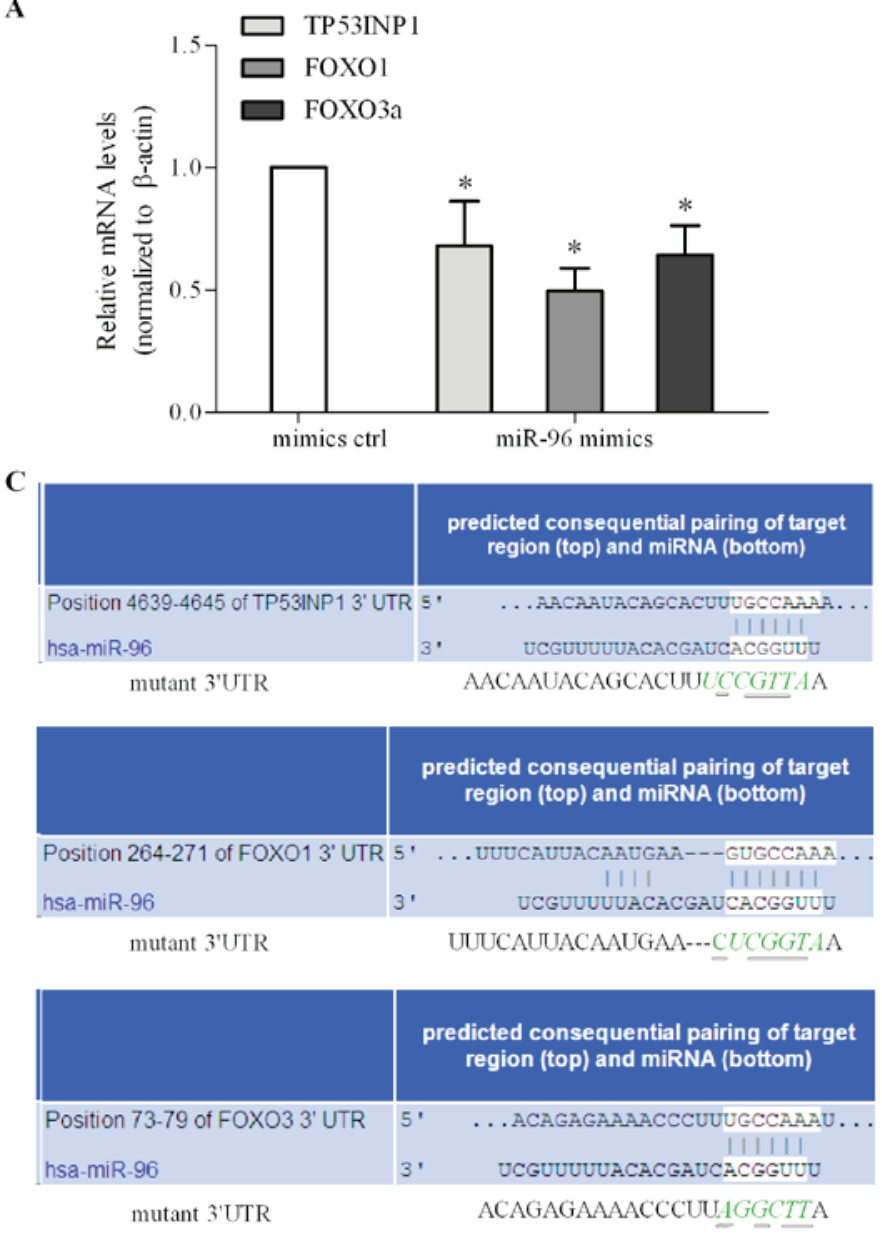

B

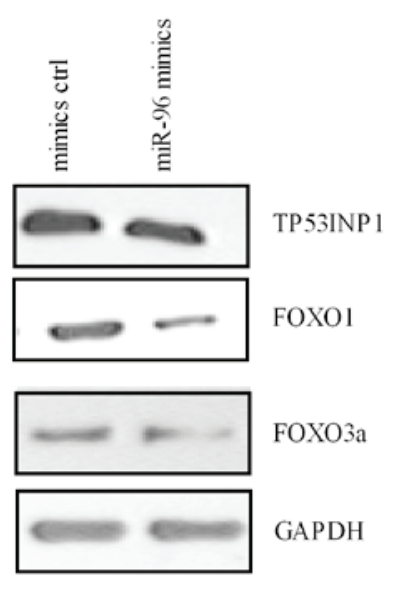

D

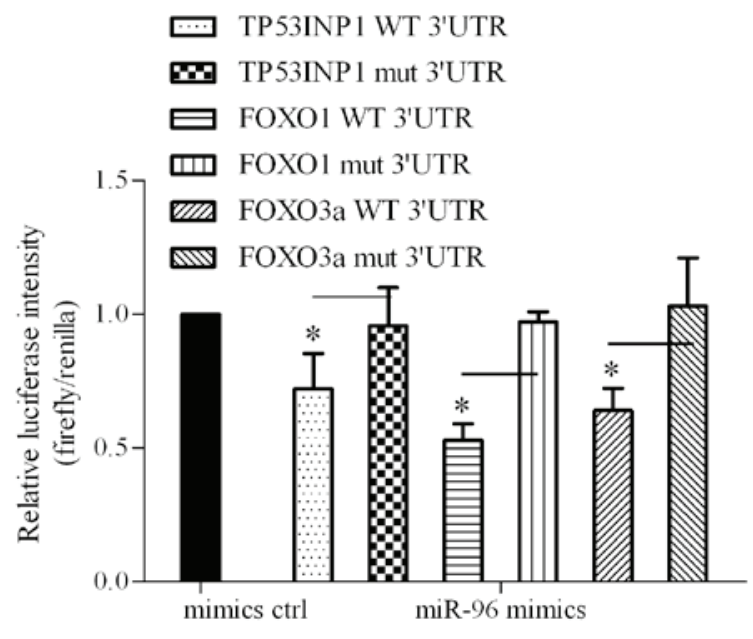

Figure 4. TP53INP1, FOXO1 and FOXO3a are direct targets of miR-96. (A) Cells were transfected with miR-96 mimics or control and harvested for RNA isolation to analyze the effect of miR-96 on TP53INP1, FOXO1 and FOXO3a mRNA expression levels by reverse transcription quantitative polymerase chain reaction. $\beta$-actin was used as an internal control. (B) Transfected cells were subjected to western blot analysis to determine protein expression levels of TP53INP1, FOXO1 and FOXO3a. GAPDH was used as an internal control. (C) Alignments between miR-96 and the binding sites in the sequences at the 3'UTR. A mutation within the binding sites was generated. The asterisks represent the mutated nucleotides. (D) Cells were co-transfected with miR-96 and the WT or mutant 3'UTR and subjected to luciferase reporter assay for the analysis of the effect of miR-96 on the intensity controlled by the 3'UTR. Data were from three independent experiments and values are expressed as the mean \pm standard deviation. ${ }^{*} \mathrm{P}<0.05$ vs. control. TP53INP1, tumor protein $\mathrm{p} 53$ inducible nuclear protein 1; FOXO1, forkhead box protein O1; miRNA, microRNA; miR-96, microRNA-96; WT, wild-type; mut, mutant; hsa, Homo sapiens.

miR-96 directly regulates TP53INP1, FOXO1 and FOXO3a expression by targeting their 3'UTR. Two algorithms, miRanda (http://www.microrna.org/microrna/home.do) and TargetScan (http://www.targetscan.org/), were used for the prediction of miR-96 candidate targets. Based on their potential roles in cell growth and the miR-96 binding sites located in their target mRNA 3'UTR sequences, TP53INP1, FOXO1 and FOXO3a were selected as candidate targets for investigation. Previous studies reported that FOXO1 and FOXO3a were direct targets of miR-96 in prostate and breast cancer and were correlated with cellular proliferation (17-19). However, the association between miR-96 and FOXO1/FOXO3a remain to be elucidated. In the present study, the roles of miR-96 in TP53INP1, FOXO1 and FOXO3 expression were examined. As indicated in Fig. 4A, miR-96 inhibited the mRNA expression of TP53INP1, FOXO1 and FOXO3a by 230,3 and $50 \%$, respectively. Analogous to the effect of miR-96 on mRNA expression levels, miR-96 also inhibited protein expression levels of the genes evaluated (Fig. 4B).

To investigate the direct regulatory roles of miR-96 in these genes, the 3'UTR-containing miR-96 binding sites (Fig. 4C) were cloned downstream of the luciferase reporter gene. The cells were subsequently co-transfected with miR-96 and the cloned luciferase reporter. As indicated in Fig. 4D, it was observed that miR-96 significantly inhibited luciferase intensity of the 3'UTR of TP53INP1, FOXO1 and FOXO3a. To further confirm that these binding sites mediated the inhibitory function of miR-96, mutations within the binding sites were generated. It was demonstrated that miR-96 did not influence the fluorescence intensity of TP53INP1, FOXO1 and FOXO3a containing the mutant 3'UTR (Fig. 4D). These results suggested that TP53INP1, FOXO1 and FOXO3a were direct target genes of miR-96 and were negatively regulated by miR-96. 


\section{Discussion}

miRNAs function as key regulators of gene expression involved in diverse biological processes. A significant body of evidence demonstrates that abnormal miRNA expression is closely associated with the pathogenesis of numerous types of cancer $(11,12)$. Therefore, determination of the differentially expressed miRNAs is crucial for elucidation of the regulatory mechanisms of miRNAs in cancer carcinogenesis. Previous studies have screened miRNAs using microarray and RT-qPCR for the comparison of expression levels in CRC and those of paired non-cancerous tissues $(11,12)$, and have indicated that miR-96 was significantly upregulated in CRC tissues. miR-96 was demonstrated to promote hepatocellular cell proliferation and colony formation (20). Studies have indicated that in prostate cancer, miR-96 upregulation contributes to cell proliferation and reduces cell apoptosis by targeting FOXO1 (17,21). miR-96 also enhances cell proliferation of breast cancer by targeting FOXO1 and FOXO3a $(18,19)$. These results suggested that miR-96 was linked to tumor growth and carcinogenesis, functioning as an oncogene. In agreement with the results of these previous studies, the present study revealed that miR-96 was overexpressed in CRC tissues, compared to expression levels in adjacent normal tissues. Furthermore, miR-96 promoted CRC cell viability, colony formation and cell cycle progression, while inhibition of miR-96 suppressed cell proliferation, which indicated that miR-96 functioned as an oncogenic miRNA.

There is disagreement regarding the potential roles of miR-96 in diverse types of cancer. In pancreatic cancer, miR-96 was reported to be downregulated and to suppress cell proliferation, tumor growth and metastasis-associated characteristics by targeting the oncogene $K R A S$, and therefore functioning as a tumor suppressor (22). The opposing roles of miR-96 in cancer pathogenesis may be associated with its target genes. This phenomenon has been reported in the regulatory effects exerted by other miRNAs (23). For example, miR-125b was downregulated in squamous cell carcinoma and suppressed cell proliferation, migration and invasion by targeting matrix metallopeptidase 13, therefore functioning as a tumor suppressor (23). However, miR-125b was reported to contribute to cell proliferation and migration by the suppression of tumor suppressor TP53INP1 in type II endometrial carcinoma cells (24). These data suggested that the effects of miRNAs may be cell- or tissue-specific and the specific target genes mediate their functions in disease pathogenesis. Therefore, in the present study, the targets of miR-96 in CRC cells were identified, which may aid in the elucidation of the regulatory mechanisms of miR-96 in CRC.

TP53INP1 is a p53 target gene and mediates the roles of p53 in the suppression of cell growth and induction of cell apoptosis, functioning as a tumor suppressor (25). TP53INP1 was reported to be downregulated in diverse types of cancer and the decreased expression of TP53INP1 was shown to contribute to the pathogenesis of cancer (26-28). TP53INP1 therefore has potential as a therapeutic target for cancers. In the present study, it was demonstrated that the TP53INP1 3'UTR contained conserved binding sites for miR-96. RT-qPCR and western blot analysis indicated that miR-96 inhibited TP53INP1 mRNA and protein expression. A luciferase reporter assay was used to determine whether miR-96 directly targeted the TP53INP1 3'UTR. The results suggested that miR-96 suppressed TP53INP1 3'UTR fluorescence intensity, whereas miR-96 had no effect on the mutant TP53INP1 3'UTR. These data suggested that miR-96 directly suppressed TP53INP1 by binding to sequences in the 3'UTR of TP53INP1.

In addition, two target genes of miR-96, FOXO1 and FOXO3a, were evaluated. Previous studies have indicated that FOXO1 and FOXO3a are target genes of miR-96 in prostate and breast cancer, respectively $(17,18)$. FOXO1 and FOXO3a are forkhead transcription factors of the $\mathrm{O}$ class, involved in cell survival, apoptosis and the suppression of tumor growth $(29,30)$. The results of the present study indicated that ectopic miR-96 expression resulted in the suppression of FOXO1 and FOXO3a mRNA and protein expression levels. The results of the luciferase reporter assay indicated that luciferase intensity was controlled by the FOXO1 and FOXO3a 3'UTRs and once the 3'UTR binding sites of miR-96 were mutated, miR-96 exerted no effect on the fluorescence intensity. These data indicated that FOXO1 and FOXO3a were target genes for miR-96 in CRC. Considering the roles of TP53INP1, FOXO1 and FOXO3a and also the regulation of their expression by miR-96, it was hypothesized that these genes mediated the functions of miR-96 in CRC cell growth and proliferation. Further study is required in order to elucidate the therapeutic potential of these genes in CRC.

In conclusion, the results of the present study indicated that miR-96 was upregulated in CRC and promoted cell growth and proliferation, functioning as an oncogene. In addition, the direct target genes of miR-96 in CRC, TP53INP1, FOXO1 and FOXO3a were identified, which may aid in the elucidation of the regulatory mechanisms of miR-96 in CRC carcinogenesis. These results suggested that miR-96 may act as a biomarker for CRC diagnosis and that it has potential as an miRNA-based therapeutic target for the treatment of CRC. Future studies should focus on in vivo investigation of the functions of miR-96 in CRC in order to provide more beneficial evidence for the clinical application of miRNAs.

\section{References}

1. Bartel DP: MicroRNAs: genomics, biogenesis, mechanism, and function. Cell 116: 281-297, 2004.

2. Brennecke J, Stark A, Russell RB and Cohen SM: Principles of microRNA-target recognition. PLoS Biol 3: e85, 2005.

3. Ambros V: The functions of animal microRNAs. Nature 431: 350-355, 2004.

4. Calin GA, Sevignani C, Dumitru CD, et al: Human microRNA genes are frequently located at fragile sites and genomic regions involved in cancers. Proc Natl Acad Sci USA 101: 2999-3004, 2004.

5. Nassirpour R, Mehta PP and Yin MJ: miR-122 regulates tumorigenesis in hepatocellular carcinoma by targeting AKT3. PLoS One 8: e79655, 2013.

6. Gaziel-Sovran A, Segura MF, Di Micco R, et al: miR-30b/30d regulation of GalNAc transferases enhances invasion and immunosuppression during metastasis. Cancer Cell 20: 104-118, 2011.

7. Fearon ER: Molecular genetics of colorectal cancer. Annu Rev Pathol 6: 479-507, 2011.

8. Kanthan R, Senger JL and Kanthan SC: Molecular events in primary and metastatic colorectal carcinoma: a review. Patholog Res Int 2012: 597497, 2012.

9. Kaemmerer E, Klaus C, Jeon MK and Gassler N: Molecular classification of colorectal carcinomas: The genotype-to-phenotype relation. World J Gastroenterol 19: 8163-8167, 2013.

10. Siegel R, Naishadham D and Jemal A: Cancer statistics, 2013. CA Cancer J Clin 63: 11-30, 2013. 
11. Sarver AL, French AJ, Borralho PM, et al: Human colon cancer profiles show differential microRNA expression depending on mismatch repair status and are characteristic of undifferentiated proliferative states. BMC Cancer 9: 401, 2009.

12. Bandres E, Cubedo E, Agirre X, et al: Identification by real-time PCR of 13 mature microRNAs differentially expressed in colorectal cancer and non-tumoral tissues. Mol Cancer 5: 29, 2006.

13. Sarver AL, Li L and Subramanian S: MicroRNA miR-183 functions as an oncogene by targeting the transcription factor EGR1 and promoting tumor cell migration. Cancer Res 70: 9570-9580, 2010.

14. Khatri R and Subramanian S: MicroRNA-135b and its circuitry networks as potential therapeutic targets in colon cancer. Front Oncol 3: 268, 2013.

15. Cekaite L, Rantala JK, Bruun J, et al: MiR-9, -31, and -182 deregulation promote proliferation and tumor cell survival in colon cancer. Neoplasia 14: 868-879, 2012.

16. Chen C, Ridzon DA, Broomer AJ, et al: Real-time quantification of microRNAs by stem-loop RT-PCR. Nucleic Acids Res 33: e179, 2005.

17. Haflidadottir BS, Larne O, Martin M, et al: Upregulation of miR-96 enhances cellular proliferation of prostate cancer cells through FOXO1. PLoS One 8: e72400, 2013.

18. Lin H, Dai T, Xiong H, et al: Unregulated miR-96 induces cell proliferation in human breast cancer by downregulating transcriptional factor FOXO3a. PLoS One 5: e15797, 2010

19. Guttilla IK and White BA: Coordinate regulation of FOXO1 by miR-27a, miR-96, and miR-182 in breast cancer cells. J Biol Chem 284: 23204-23216, 2009.

20. Xu D, He X, Chang Y, et al: Inhibition of miR-96 expression reduces cell proliferation and clonogenicity of HepG2 hepatoma cells. Oncol Rep 29: 653-661, 2013.
21. Fendler A, Jung M, Stephan C, et al: The antiapoptotic function of miR-96 in prostate cancer by inhibition of FOXO1. PLoS One 8: e80807, 2013

22. Yu S, Lu Z, Liu C, et al: miRNA-96 suppresses KRAS and functions as a tumor suppressor gene in pancreatic cancer. Cancer Res 70: 6015-6025, 2010.

23. Xu N, Zhang L, Meisgen F, et al: MicroRNA-125b down-regulates matrix metallopeptidase 13 and inhibits cutaneous squamous cell carcinoma cell proliferation, migration, and invasion. J Biol Chem 287: 29899-29908, 2012.

24. Jiang F, Liu T, He Y, et al: MiR-125b promotes proliferation and migration of type II endometrial carcinoma cells through targeting TP53INP1 tumor suppressor in vitro and in vivo. BMC Cancer 11: 425, 2011.

25. Zhang CM, Zhao J and Deng HY: MiR-155 promotes proliferation of human breast cancer MCF-7 cells through targeting tumor protein 53-induced nuclear protein 1. J Biomed Sci 20: 79, 2013.

26. Jiang PH, Motoo Y, Garcia S, et al: Down-expression of tumor protein p53-induced nuclear protein 1 in human gastric cancer. World J Gastroenterol 12: 691-696, 2006.

27. Shahbazi J, Lock R and Liu T: Tumor protein 53-induced nuclear protein 1 enhances p53 function and represses tumorigenesis. Front Genet 4: 80, 2013

28. Ito $\mathrm{Y}$, Motoo $\mathrm{Y}$, Yoshida $\mathrm{H}$, et al: Decreased expression of tumor protein p53-induced nuclear protein 1 (TP53INP1) in breast carcinoma. Anticancer Res 26: 4391-4395, 2006.

29. Maiese K, Chong ZZ, Shang YC and Hou J: Clever cancer strategies with FoxO transcription factors. Cell Cycle 7: 3829-3839, 2008.

30. Guo JP, Tian W, Shu S, et al: IKBKE phosphorylation and inhibition of FOXO3a: a mechanism of IKBKE oncogenic function. PLoS One 8: e63636, 2013. 